Revue d'histoire de l'Amérique française

78. REVUE D.HISTOIRE DE L'AMÉRIQUE FRANÇAISE

\title{
L'histoire urbaine au Québec : la recherche récente à la maîtrise et au doctorat
}

\section{François Guérard}

Volume 54, numéro 2, automne 2000

URI : https://id.erudit.org/iderudit/005458ar

DOI : https://doi.org/10.7202/005458ar

Aller au sommaire du numéro

Éditeur(s)

Institut d'histoire de l'Amérique française

ISSN

0035-2357 (imprimé)

1492-1383 (numérique)

Découvrir la revue

Citer cet article

Guérard, F. (2000). L'histoire urbaine au Québec : la recherche récente à la maîtrise et au doctorat. Revue d'histoire de l'Amérique française, 54(2), 247-268. https://doi.org/10.7202/005458ar
Résumé de l'article

L'auteur cherche à dégager les grandes orientations qui ont marqué le champ de l'histoire urbaine au Québec depuis une décennie, à travers une recension critique des mémoires de maîtrise et des thèses de doctorat. Quelles perspectives ont été privilégiées ? Discerne-t-on quelque renouveau ? Il apparaît, dans l'ensemble, que nombre de travaux d'étudiants ont largement contribué à approfondir nos connaissances sur l'urbain au Québec. Toutefois, on peut souhaiter un raffermissement des pratiques interdisciplinaires qui tirerait exemple de l'heureux mariage de la géographie et de l'histoire, ainsi que l'ajout d'études qui embrasseraient des territoires et des périodes d'enquête plus vastes. 


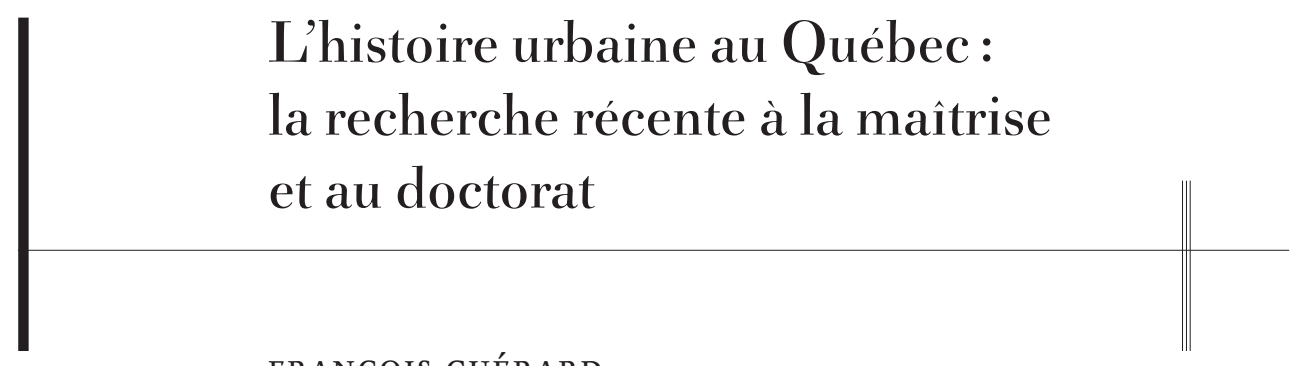

FRANÇOIS GUÉRARD

Centre interuniversitaire d'études québécoises

Université du Québec à Trois-Rivières

RÉsumÉ - L'auteur cherche à dégager les grandes orientations qui ont marqué le champ de l'histoire urbaine au Québec depuis une décennie, à travers une recension critique des mémoires de maîtrise et des thèses de doctorat. Quelles perspectives ont été privilégiées? Discerne-t-on quelque renouveau? II apparaît, dans l'ensemble, que nombre de travaux d'étudiants ont largement contribué à approfondir nos connaissances sur l'urbain au Québec. Toutefois, on peut souhaiter un raffermissement des pratiques interdisciplinaires qui tirerait exemple de l'heureux mariage de la géographie et de l'histoire, ainsi que l'ajout d'études qui embrasseraient des territoires et des périodes d'enquête plus vastes.

ABSTRACT - The author highlights the major orientations in Quebec urban history during the last decade by reviewing doctoral and master's dissertations. Which perspectives dominated this field of research? Are there new ways to deal with it? It appears that student's works largely contributed to enhance our knowledge. Yet, the author thinks that a better understanding would have resulted from the strenghtening of interdisciplinary practices inspired by the example of geography and history, and from carrying out studies spanning broader territories and periods.

$\mathrm{n}$ ne s'est pas souvent arrêté à dégager les centres de gravité et les
points faibles du champ d'étude que constitue l'histoire urbaine au Québec ${ }^{1}$. C’est ce qui sera tenté ici, pour les dernières dix années, en

1. Paul-André Linteau et Alan F. J. Artibise ont fait, il y a quinze ans, un tel tour d'horizon pour le Canada. Voir P.-A. Linteau et Alan F. J. Artibise, L'évolution de l'urbanisation au Canada : une analyse des perspectives et des interprétations (Winnipeg, The Institute of Urban Studies, University of Winnipeg, rapport $n^{\circ} 5,1984$ ). 
circonscrivant la réflexion aux thèses de doctorat et aux mémoires de maîtrise qui ont mené à l'obtention d'un diplôme depuis 1989. Ces ouvrages témoignent éloquemment de l'évolution des préoccupations à la fois des étudiants et des professeurs qui ont supervisé leur démarche. Dans certains cas, ils reposent d'ailleurs sur des recherches que les étudiants ont menées dans le cadre de chantiers plus vastes pilotés par leurs directeurs de mémoire ou de thèse. Aussi livrent-ils un portrait certes incomplet, mais assez fidèle des questionnements dominants.

Tout bilan historiographique sur l'histoire urbaine, tout guide bibliographique se heurte dès l'abord à la difficulté de définir ce champ d'études ${ }^{2}$. Les auteurs de tels travaux choisissent souvent d'embrasser une conception élargie du champ, non sans se montrer critiques à son égard. Ils font preuve ainsi d'un pragmatisme qui leur permet d'intégrer des études portant sur des aspects diversifiés de la vie urbaine, tout en évitant de revendiquer pour l'ensemble de ces textes une appartenance claire à l'histoire urbaine. En fait, bien souvent, bon nombre des études ainsi recensées appartiennent tout autant, sinon plus, à d'autres champs de recherche.

Notre démarche de sélection des mémoires et des thèses devait tenir compte de l'imprécision des frontières de l'histoire urbaine. Une sélection préliminaire a d'abord été effectuée qui englobait un champ d'études délibérément bien plus vaste que celui à couvrir. Tous les titres qui, de près ou de loin, semblent concerner la ville et l'urbain au Québec, tous ceux qui laissent entrevoir un intérêt pour l'histoire de la ville ou pour l'histoire de tout autre thème (quel qu'il soit) dans la ville, ont été recueillis. Lors du repérage des mémoires et des thèses, nous avons en outre cherché à couvrir l'ensemble des universités et des programmes susceptibles de produire de tels travaux en histoire urbaine. Cela vaut pour les programmes d'histoire, bien sûr, mais aussi de géographie, d'urbanisme et d'aménagement, d'architecture, d'archéologie, de sociologie, de démographie, d'études littéraires, d'histoire de l'art... Le premier outil bibliographique consulté recense annuellement les thèses et les mémoires

2. Voir, par exemple, Richard Rodger, Research in Urban History. A Classified Survey of Doctoral and Masters' Theses (Aldershot, Angleterre, Scolar Press, 1994); Isabelle Backouche, L'histoire urbaine en France (Moyen-Âge - Xx siècle). Guide bibliographique 1965-1996 (Paris/Montréal, L'Harmattan, 1998); A. F. J. Artibise et Gilbert A. Stelter, Canada's Urban Past : A Bibliography to 1980 and Guide to Canadian Urban Studies (Vancouver, University of British Columbia Press, 1981); S. M. Blooming, "City Limits. Two Decades of Urban History in JUH», Journal of Urban History, 21,1 (novembre 1994): 7-30. 
en histoire canadienne ${ }^{3}$. D’autres outils ${ }^{4}$, principalement les répertoires informatisés des bibliothèques universitaires du Québec et un fichier informatisé des résumés de mémoires et de thèses nord-américains ${ }^{5}$, ont permis d'élargir encore la banque de titres. Un fichier de plus de deux cents références est résulté de cette première étape.

Les mémoires et les thèses repérés ont ensuite été consultés afin de choisir ceux qui formeraient notre corpus. De prime abord, aucun thème ou objet de recherche n'a été exclu : de notre point de vue, la spécificité du champ de l'histoire urbaine ne réside pas dans une liste de thèmes ou d'objets qui lui seraient propres ou en seraient d'office bannis. Certains, certes, lui appartiennent d'emblée : par exemple, l'urbanisation, la formation du réseau urbain et les rapports entretenus par la ville avec l'extérieur; la production de l'espace urbain et la morphologie urbaine; l'activité des municipalités et celle des élites urbaines visant à promouvoir le développement de leur ville ou à aménager les services offerts aux citadins; ou encore les phénomènes culturels propres à la ville. Il s'agit là du noyau dur de l'histoire urbaine.

Au-delà de ce noyau, l'histoire urbaine peut couvrir quantités de thèmes ou d'objets de recherche, pour autant que leur traitement respecte ce qui confère au champ sa spécificité. Celle-ci relève, à notre avis, du regard même que l'historien pose sur l'objet de ses recherches, plus précisément d'une démarche intellectuelle où le fait urbain est perçu comme une catégorie d'étude signifiante et utilisé de la sorte. Aussi, pour qu'un texte soit retenu dans notre sélection finale, sa problématique devait renfermer une préoccupation importante pour le fait urbain, et la ville s'y avérer partie prenante de l'interprétation, plutôt que simple toile de fond ou localisation propice à l'examen de tel ou tel phénomène. Le corpus dont il sera question ici ne recouvre donc pas tout ce qui se passe dans la ville, loin s'en faut. À l'inverse, il comporte une ouverture sur des textes qui

3. Société historique du Canada, Répertoire des thèses en cours portant sur des sujets d'histoire et autres sujets connexes, années concernées.

4. Toutes ces sources présentent le défaut de ne pas recenser les titres de la dernière année. Nous avons effectué quelques vérifications dans certains départements, sans toutefois systématiser cette démarche. Michèle Dagenais, par exemple, nous a fait parvenir une liste complète pour le département d'histoire de l'Université de Montréal, ce dont nous la remercions. Celui de l’Université du Québec à Montréal distribue périodiquement des comptes rendus de ses activités, ce qui a été également utile.

5. University Microfilms International, Dissertation abstracts [document électronique] (Ann Arbor, Mich., University Microfilms International). 
situeraient leur territoire d'enquête hors de la ville, mais dont la problématique accorderait une place significative au phénomène urbain.

Toute une série de travaux (identifiés dans les notes infrapaginales), bien qu'ils enrichissent nos connaissances sur la société urbaine, ont été éliminés. Il en va ainsi, en général, de ceux qui portent sur des institutions comme les hôpitaux ${ }^{6}$ et les services sociaux ou correctionnels ${ }^{7}$, de même que sur des groupes socioprofessionnels. Bon nombre d'étudiants se sont ainsi penchés sur les mondes artisanal ${ }^{8}$ ou marchand $^{9}$, quelques-uns sur

6. Pour la recherche sur les hôpitaux, voir : François Guérard, «Ville et santé au Québec : un bilan de la recherche historique», Revue d'histoire de l'Amérique française, 53,1 (été 1999) : 19-45.

7. Sur les services sociaux et correctionnels, voir : Lucie Bonnier, L'entraide au quotidien : l'exemple de la paroisse Sainte-Brigide, Montréal, 1930-1945, mémoire de maîtrise (histoire), Université du Québec à Montréal 1996; Danielle Choquette, Histoire de l'Institut de réadaptation de Montréal de 1949 à 1976, mémoire de maîtrise (histoire), Université de Montréal, 1996; Sylvie Ménard, L'Institut Saint-Antoine et la problématique de réforme des garçons délinquants au Québec (1873-1909), thèse de doctorat (histoire) Université du Québec à Montréal, 1998; Rachelle Pelletier, Le genre de la pauvreté : l'assistance aux indigents à Sherbrooke de 1922 à 1940, mémoire de maîtrise (sciences humaines/histoire), Université de Sherbrooke, 1994; Stéphane-Doréus Perreault, Les Clercs de Saint-Viateur et l'Institution des sourds-muets, 1848-1930 : berceau de la communauté sourde montréalaise, mémoire de maîtrise (histoire), Université d’Ottawa, 1996; Ann Kirsten Perry, Manliness, Goodness, and God: Poverty, Gender, and Social Reform in English-Speaking Montreal, 1890-1929, mémoire de maîtrise (histoire), Queen's University, 1998; Pierre-Georges Roy, L'orphelinat SaintArsène, de 1906-1925, mémoire de maîtrise (histoire), Université de Montréal, 1990.

8. Voir Peter Bischoff, Tensions et solidarité : la formation des traditions syndicales chez les mouleurs de Montréal, Hamilton et Toronto, 1851 à 1893, thèse de doctorat (histoire), Université de Montréal, 1992; Dominique Bouchard, Le niveau de vie des artisans du fer à Québec et à Montréal entre 1730 et 1780, mémoire de maîtrise (histoire), Université de Montréal, 1992; Luc Bouffard, Étude socioprofessionnelle des familles cordonnières du quartier Saint-Jacques, à Montréal, entre 1861 et 1891, mémoire de maîtrise (histoire), Université de Montréal, 1997; Jean-Francois Cardin, Travailleurs industriels et syndicalisme en période de prospérité, conditions de travail, conditions de vie et conscience ouvrière des métallos montréalais durant la guerre et l'après-guerre (1940-1960), thèse de doctorat (histoire), Université de Montréal, 1992; Josée Desbiens, Le niveau de vie et l'univers domestique des artisans montréalais entre 1740 et 1809, mémoire de maitrise (histoire), Université de Montréal, 1991 ; Robert Lamonde, Les boulangers de Montréal, de la fondation à 1750 : étude d'histoire socio-économique, mémoire de maitrise (histoire), Université de Montréal, 1997; France-Isabelle Langlois, Familles de charpentiers et de menuisiers à Montréal au XVIII siècle : alliances matrimoniales et reproduction sociale, mémoire de maitrise (histoire), Université de Montréal, 1996; Dominique Laperle, Les meuniers de la région de Montréal à l'époque de la Nouvelle-France (1642-1760) : alliances matrimoniales et reproduction sociale, mémoire de maîtrise (histoire), Université de Montréal, 1997; Robert Tremblay, Du forgeron au machiniste : l'impact social de la mécanisation des opérations d'usinage dans l'industrie de la métallurgie à Montréal de 1815 à 1860, thèse de doctorat (histoire), Université du Québec à Montréal, 1992.

9. George Bervin, Les marchands-négociants et la diversité de leurs activités économiques à Québec entre 1800 et 1825, thèse de doctorat (histoire), Université de Montréal, 1989; Marcelle Cinq-Mars, Représentations et stratégies sociales d'un étranger à Québec à la fin du XVIII siècle : analyse du journal 
d'autres occupations ${ }^{10}$. Certains de ces travaux, certes, comportent des éléments de problématique spécifiquement urbains. En règle générale, toutefois, la ville n'y constitue essentiellement qu'un cadre d'étude. Plus que de l'histoire urbaine, la plupart de ces mémoires et de ces thèses relèvent, nous paraît-il, d'autres champs de recherche, comme l'histoire des travailleurs, et ce même lorsque le groupe étudié s'avère typiquement urbain.

De la même façon, les études consacrées aux groupes religieux ou ethniques ont été écartées ${ }^{11}$. Il importe de souligner ici que d'autres chercheurs auraient pu effectuer un choix différent. Ces groupes, en effet, ont joué un rôle majeur dans l'histoire d'une ville comme Montréal ${ }^{12}$. De plus, l'histoire urbaine nord-américaine comporte un volet assez important

personnel du marchand Johann Henrich Juncken (septembre 1788-mai 1789), mémoire de maîtrise (histoire), Université Laval, 1990; François Gagnon, Marchands voyageurs et équipeurs de Montréal, 1715-1750, mémoire de maîtrise (histoire), Université de Montréal, 1995 ; Linda Kerr, Quebec : The Making of an Imperial Mercantile Community, 1760-1768, thèse de doctorat (histoire), University of Alberta, 1992; Kathryn Agnes Young, Kin, Commerce, and Community : Merchants in the Port of Quebec from 1717 to 1745, thèse de doctorat (histoire), Université du Manitoba, 1991.

10. Voir Grace Laing Hogg, The Legal Rights of Masters, Mistresses and Domestic Servants in Montreal, 1816-1829, mémoire de maîtrise (histoire), Université McGill, 1989 ; Dominique Marquis, Les avocats dans la société montréalaise au tournant du xx siècle, mémoire de maîtrise (histoire), Université du Québec à Montréal, 1989; Marie-Claude Poliquin, Les aubergistes et les cabaretiers montréalais entre 1700 et 1755, mémoire de maîtrise (histoire), Université McGill, 1996; Christine Veilleux, Les gens de justice à Québec, 1760-1867, thèse de doctorat (histoire), Université Laval, 1990.

11. Voir Cynthia S. Fish, Images and Reality of Fatherhood: A Case Study of Montreal's Protestant Middle Class, 1870-1914, thèse de doctorat (histoire), Université McGill, 1991; Sarah Filotas, Les organisations féminines de la communauté juive montréalaise, 1918-1948, mémoire de maîtrise (histoire), Université de Montréal, 1998; Yves Carmelo Luciano Gadler, The Education of Italians in Montreal, 1895 to 1960, mémoire de maîtrise (administration et études des politiques), Université McGill, 1994 ; Jan Grabowski, The Common Ground Settled Natives and French in Montreal 1667-1760, thèse de doctorat (histoire), Université de Montréal, 1993; Edna Lévesque, Les mécanismes privés d'accueil et d'assistance aux immigrants : une étude de cas : la société d'assistance aux immigrants : 19501957, mémoire de maîtrise (histoire), Université du Québec à Montréal, 1991; Meenakshi Gupta, Les entrepreneurs indiens à Montréal, de 1967 à nos jours/The Indian Business Community in Montreal. From 1967 to Present, mémoire de maîtrise (études québécoises), Université du Québec à TroisRivières, 1995 ; Norman Joseph Marino, The Antiochian Orthodox Syrians of Montreal, 1905-1980 : A Historical Study of Cultural and Social Change Over Three Generations, mémoire de maîtrise (histoire), Université Concordia, 1994; Martine Rodrigue, Les Franco-Américains à Montréal au début du XX siècle, mémoire de maîtrise (histoire), Université du Québec à Montréal, 1997; Arlette Siboni Corcos, Montréal, les Juifs et l'école, thèse de doctorat (éducation comparée), Université de Montréal, 1995 ; Isabelle Soucy, La famille ouvrière juive à Montréal au tournant du Xxe siècle, mémoire de maîtrise (histoire), Université du Québec à Montréal.

12. Paul-André Linteau, notamment, a mis ce point en lumière : P.-A. Linteau, Histoire de Montréal depuis la Confédération (Montréal, Boréal, 1992). 
d'études ethniques qui ajoutent à notre compréhension de la société urbaine. Enfin, la recherche sur la formation et l'évolution de la société urbaine s'est enrichie au cours de la dernière décennie de réflexions sur les groupes sociaux dans une perspective identitaire ${ }^{13}$. Quoi qu'il en soit, le bloc de mémoires et de thèses qui appartiennent à ce volet ne sera pas commenté ici, dans la mesure où leurs auteurs n'ont généralement pas conféré à leur problématique une dimension spécifiquement urbaine.

On le voit, notre perception du champ de l'histoire urbaine tend à y restreindre l'annexion de territoires adjacents, et ce, bien qu'elle mette de l'avant un type de démarche plutôt qu'une liste plus ou moins ouverte d'objets ou de thèmes qui lui appartiendraient. Nous estimons en fait que les frontières de ce champ, quoique nécessairement poreuses et imprécises, ne sont pas pour autant si aisément extensibles.

Pas moins de 20 thèses et 50 mémoires ont été finalement retenus. Deux des universités d'où ils proviennent se démarquent par une production particulièrement abondante : l'Université du Québec à Montréal (UQAM) et l'Université Laval. Pour l'UQAM, cela relève d'une tradition bien établie : depuis plus de 25 ans, son département d'histoire s'est imposé comme un chef de file dans le champ de l'histoire urbaine au Québec, en particulier à travers les activités du Groupe de recherche sur l'histoire de Montréal (GRHM) ${ }^{14}$. À l'Université Laval, les nombreux travaux recensés proviennent de plusieurs programmes. Quatre autres établissements ressortent : l'Université de Montréal, l'Université du Québec à Trois-Rivières (UQTR), avec le Centre interuniversitaire d'études québécoises, l'Université McGill, où loge le Montreal History Group, et l'Université de Sherbrooke ${ }^{15}$.

13. Claire Poitras, dans une communication présentée au congrès annuel de l'Institut d'histoire de l'Amérique française en 1999, a souligné l'émergence de la question identitaire dans les articles de revues publiés depuis dix ans dans le champ de l'histoire urbaine : «L'histoire urbaine au Québec durant les années 1990 : de nouvelles tendances?». Voir l'article du même titre dans le présent numéro.

14. Il existe un compte rendu du travail effectué depuis 1972 par le Groupe de recherche sur l'histoire de Montréal : P.-A. Linteau et al., 25 ans d'histoire de Montréal. Le Groupe de recherche sur l'histoire de Montréal 1972-1997 (Montréal, département d'histoire, Université du Québec à Montréal, 1998).

15. Notons que nous n'avons pas eu en main les mémoires rédigés dans le cadre d'un programme d'échanges entre l'Université du Québec à Montréal et des universités françaises. 
FIGURE 1

\section{Thèses et mémoires par université}

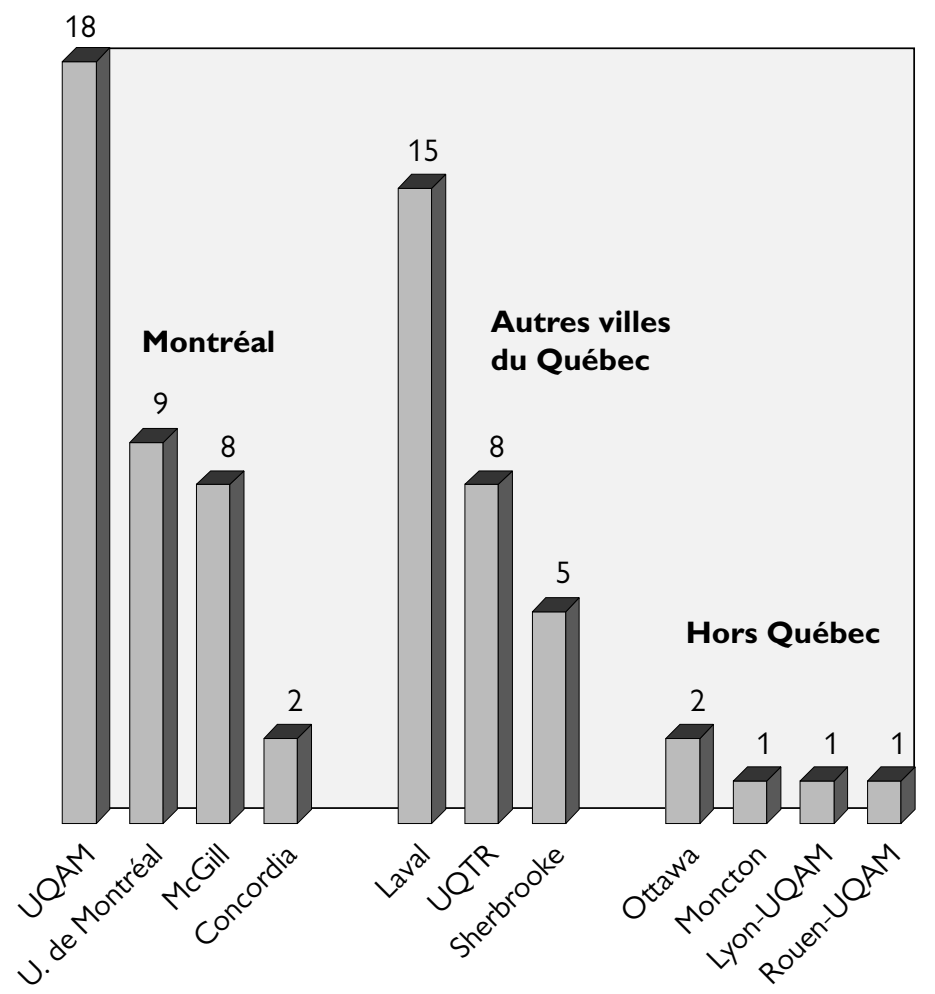

\section{DES TERRITOIRES ET DES PÉRIODES D'ENQUÊTE GÉNÉRALEMENT RESTREINTS}

La plupart des étudiants ont choisi de contenir dans des limites assez étroites l'envergure des territoires et des périodes d'enquête. Ainsi, sauf exception, leur analyse demeure confinée au territoire d'une seule ville, généralement située dans l'agglomération où loge leur université ${ }^{16}$. Les études comparatives, l'examen d'un même thème dans les contextes changeants de divers centres urbains, sont des démarches presque

16. Quatre-vingt-sept pour cent des étudiants des universités montréalaises ont situé leur objet d'études dans l'agglomération métropolitaine; à Laval, la même proportion a choisi l'agglomération de Québec (67\%) ou des villes du Bas-du-Fleuve (20\%); à Trois-Rivières, les trois quarts ont choisi des localités de la région. 
absentes du corpus. Plusieurs chercheurs, pourtant, ont souhaité de telles études, et ce depuis de nombreuses années.

Le cortège des villes retenues par les étudiants reflète les caractéristiques du système urbain québécois et de son évolution. L’agglomération montréalaise domine avec plus de la moitié des travaux, celle de Québec suit avec 19\%. Les $29 \%$ restants témoignent d'une ouverture sur les autres centres urbains. Toutefois, des régions comme l'Outaouais, l'AbitibiTémiscamingue, le Saguenay-Lac Saint-Jean et la Côte-Nord manquent à l'appel. En fait, les régions les plus récemment urbanisées ont été ignorées. Il faut dire que la période de loin la mieux couverte va de 1850 à 1950, avec une nette concentration autour de 1900 (figures 2 et 3) ${ }^{17}$. Il était souhaitable que cette grande phase d'urbanisation soit abondamment examinée. Toutefois, la ville préindustrielle et l'urbanisation récente, avec leur dynamisme propre, méritent plus d'attention.

Par ailleurs, les étudiants historiens ne se sont pas aventurés sur des périodes de plus de 100 ans, laissant cela à quelques-uns de leurs confrères d'autres disciplines, archéologues notamment. Personne ne s'étonnera que les mémoires ne s'établissent pas dans la longue durée, la maîtrise n'étant pas le moment de pareils exercices, mais force est de constater que les thèses ne s'y aventurent pas plus ${ }^{18}$. Il semble bien que les questions posées et les démarches pour y répondre s'accommodaient confortablement d'une certaine brièveté. Est-ce à dire que l'histoire urbaine au Québec ne s'interroge plus sur les tendances lourdes exprimées et perceptibles dans la longue durée?

Nous reviendrons sur ce point après avoir présenté le contenu thématique des mémoires et des thèses, divisé en cinq classes : l'espace relationnel des villes, les élites et le développement urbain, la ville comme entité physique et spatiale, la gestion de la ville et ses services, et enfin la société et la culture urbaines.

\section{L'ESPACE RELATIONNEL DES VILLES, UNE THÉMATIQUE DÉLAISSÉE}

La thématique intitulée «l'espace relationnel des villes» rassemble des phénomènes qui dépassent les limites de l’agglomération : rapports des villes entre elles et avec leur arrière-pays, réseaux urbains... Peu

17. Cela rejoint les constats de S. M. Blooming, op. cit.

18. Les deux tiers des études environ portent sur des périodes de 50 ans et moins, $7 \%$ seulement sur 100 ans et plus, et $27 \%$ de 51 à 100 ans. 
FIGURE 2

Mémoires et thèses selon les années incluses, aux dix ans

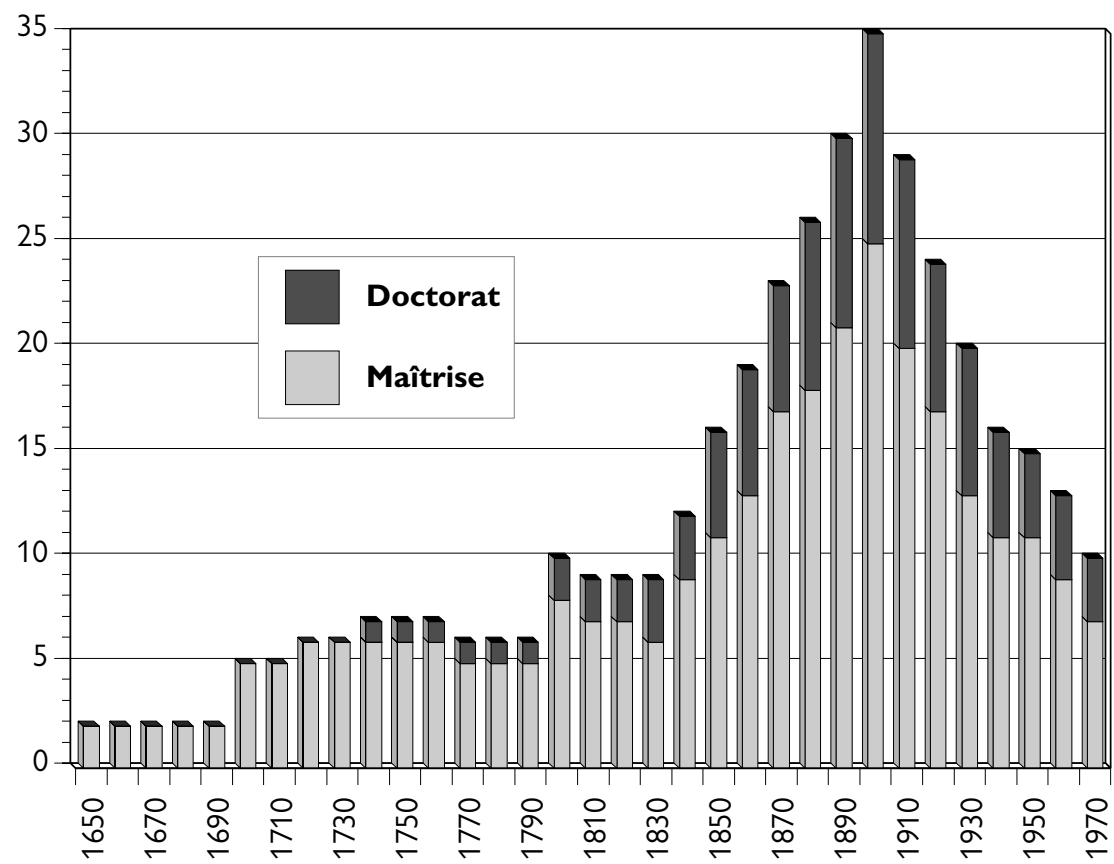

d'étudiants s'y sont intéressés. Deux ont abordé l'intense vie d'échanges qui s'établit avec le voisinage des villes en scrutant le fonctionnement des marchés montréalais au $\mathrm{XIX}^{\mathrm{e}}$ siècle ${ }^{19}$ et les transformations du village de Charlesbourg lorsqu'il devient un lieu d'échanges entre Québec et les campagnes environnantes ${ }^{20}$.

Deux autres étudiants ont mis l'accent sur l'interdépendance de la ville et de sa région. L’un argumente que Joliette doit son développement au $\mathrm{xIX}^{\mathrm{e}}$ siècle à sa fonction de services bien plus qu'à son activité industrielle ${ }^{21}$. L'autre considère que si les poussées de croissance de Coaticook sont bien liées au secteur industriel, c'est son rôle de centre régional de services qui

19. Sylvie Brouillette, Les marchés publics à Montréal : 1840-1860, mémoire de maîtrise (études québécoises), Université du Québec à Trois-Rivières, 1991.

20. Johanne Lachance, Charlesbourg, 1831-1871 : contributions à l'étude des relations villes-campagnes, mémoire de maîtrise (géographie), Université Laval, 1991.

21. Patrice Régimbald, Urbanisation et croissance urbaine : Joliette 1850-1900, mémoire de maîtrise (histoire), Université du Québec à Montréal, 1992. 
FIGURE 3

Les périodes à l'étude dans les mémoires et les thèses

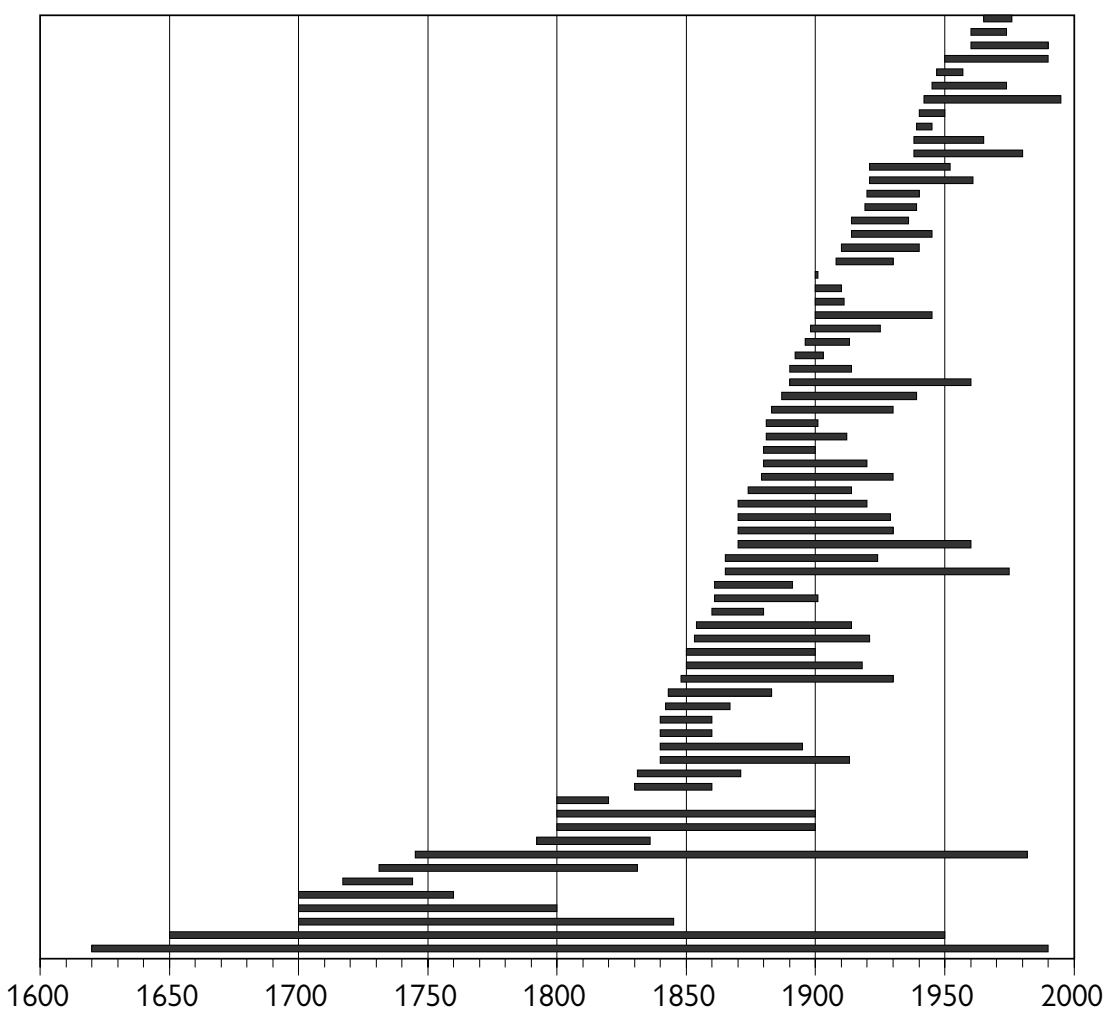

lui assure une certaine stabilité démographique en dépit des aléas de la conjoncture économique ${ }^{22}$. Comme pour d'autres petits centres étudiés, l'arrivée du rail s'avère déterminante en faisant de ces villes un lieu de transit privilégié entre la région et l'extérieur de la région, notamment en facilitant aux producteurs agricoles l'accès aux marchés extérieurs.

Une thèse, enfin, livre le seul aperçu de la décennie sur le déploiement de l'influence des principales villes sur de vastes territoires par le biais des grands réseaux de transport et d'échanges. Elle montre qu'en 1870, l'aire de relations de Québec par la voie fluviale s'étendait à l'ensemble du sys-

22. Michel Breton, La transformation de l'espace rural, l'industrialisation et les relations villecampagne entre Coaticook et les cantons de Barnston et de Barford, 1853-1921, mémoire de maîtrise (sciences humaines/histoire), Université de Sherbrooke, 1993. 
tème laurentien, avant de se contracter alors que le centre de gravité de la navigation fluviale se déplaçait vers l'ouest au profit de Montréal ${ }^{23}$. Notons que ces activités d'échange ont longtemps joué un rôle déterminant dans l'évolution de la basse-ville de Québec, ce qu'a mis en évidence une étude archéologique sur un site de la zone portuaire ${ }^{24}$.

Dans l'ensemble, le peu d'intérêt manifesté vis-à-vis du système urbain québécois en tant que réseau de relations et d'échanges déçoit quelque peu, d'autant plus qu'on abandonne ainsi une voie de recherches dont l'intérêt avait été démontré par des travaux antérieurs ${ }^{25}$. Il est vrai que ces questions relèvent pour une bonne part de l'histoire économique qui connaît des heures difficiles au Québec ${ }^{26}$.

\section{DÉVELOPPEMENT DES VILLES ET ÉLITES URBAINES : UNE THÉMATIQUE ATTRAYANTE}

D’autres étudiants se sont interrogés sur le rôle des élites urbaines dans le développement des villes, une thématique déjà bien explorée pour les grands centres, le plus souvent en reportant les questionnements des travaux antérieurs sur des villes plus petites comme Rivière-du-Loup, Montmagny, Rimouski, Trois-Rivières ou Sherbrooke ${ }^{27}$. Ils ont vérifié

23. France Normand, Pratiques et conditions de la petite navigation sur le Saint-Laurent dans les dernières décennies du XIX $x^{e}$ siècle : le cas de la batellerie du port de Québec, thèse de doctorat (études québécoises), Université du Québec à Trois-Rivières, 1995.

24. Myriam Leclerc, Appropriation de l'espace et urbanisation d'un site de la basse-ville de Québec: la première campagne de fouilles à l'îlot Hunt, mémoire de maîtrise (arts et traditions populaires), Université Laval, 1995.

25. Voir à ce sujet P.-A. Linteau et A. F. J. Artibise, op. cit. Parmi les travaux exécutés hors Québec et qui montrent bien l'intérêt de choisir comme point d'observation l'ensemble du réseau urbain, on peut consulter par exemple : Bernard Lepetit, Les villes dans la France moderne (1740-1840) (Paris, Albin Michel, 1988); Paul M. Hohenberg et Lynn Hollen Lees, La formation de l'Europe urbaine 1000-1950 (Paris, Presses universitaires de France, coll. "Histoires», 1992). Le peu d'intérêt manifesté pour les réseaux urbains a été noté aux États-Unis également, dans le Journal of Urban History : "I am particularly struck by the paucity of articles that deal explicitly with the formation and character of urban systems", S. M. Blumin, op. cit., 12.

26. Il importe de souligner qu'en France également, d'après Isabelle Backouche, dans le champ de l'histoire urbaine, «la démographie, les finances ou la question des transports semblent peu attractifs pour les chercheurs». I. Backouche, op. cit., iii.

27. Martine Côté, Industrialisation et urbanisation à Montmagny 1883-1930, mémoire de maîtrise (histoire), Université Laval, 1990; Chantal Desloges, Les propriétés foncières et résidentielles dans une ville en forte croissance : Sherbrooke 1896-1913, mémoire de maîtrise (sciences humaines/histoire), Université de Sherbrooke, 1989; Mireille Lehoux, Élus municipaux et promotion industrielle à TroisRivières, 1870-1920, mémoire de maîtrise (études québécoises), Université du Québec à TroisRivières, 1994 ; Julie Martin, Rivière-du-Loup et son espace au 19e siècle: du village linéaire à la ville industrielle, mémoire de maîtrise (histoire) Université Laval, 1994; Yves Tremblay, La participation 
dans quelle mesure le profil et l'activité des élites urbaines expliquaient le développement de la ville et surtout ses particularités. Il en ressort que ces élites considèrent la venue d'entreprises industrielles comme la clé d'un développement dont elles entendent profiter. Les milieux d'affaires ${ }^{28}$, les promoteurs immobiliers et fonciers, les autorités municipales mettent en œuvre diverses stratégies de promotion. Ces efforts, semblables d'une localité à l'autre, ne sont pas couronnés du même succès partout, par exemple en ce qui a trait aux tentatives de diversifier l'économie locale et aux politiques de subventions municipales aux entreprises.

Centrés sur les facteurs de croissance et les dynamismes locaux, ces travaux ne peuvent à eux seuls expliquer le développement différentiel des villes, conditionné en bonne partie par des facteurs externes ${ }^{29}$. Ils apportent par contre beaucoup à notre compréhension des formes que prend le développement dans des villes particulières, ainsi que de la société urbaine québécoise en braquant les projecteurs sur ceux qui ont exercé un pouvoir dans la ville.

\section{GÉRER LA VILLE, UNE QUESTION DE RAPPORTS DE POUVOIR}

Un troisième groupe de travaux concerne l'administration de la ville et des services urbains. Un bloc de textes aborde les luttes qui entourent les transformations de l'administration municipale. Parmi ceux-ci, deux mémoires portent respectivement sur un réformateur montréalais de la fin du $\mathrm{XIX}^{\mathrm{e}}$ siècle $^{30}$ et sur les institutions municipales de Grand-Mère ${ }^{31}$. La question de l'administration des municipalités de banlieues et de leur autonomie vis-à-vis de la ville-centre a également été étudiée dans les cas de Trois-Rivières-Ouest et de Shawinigan-Sud ${ }^{32}$. Une thèse a été consacrée

de l'élite locale au développement économique de Rimouski, 1890-1960, mémoire de maîtrise (histoire), Université Laval, 1989.

28. Dans le cas de Montréal, voir Louise Bouchard, Le Montreal Board of Trade : 1842-1867, mémoire de maîtrise (histoire), Université du Québec à Montréal, 1996.

29. Les effets, par exemple, de la Seconde Guerre mondiale sur le développement de Verdun ont été étudiés : Serge Marc Durflinger, City at War: The Effects of the Second World War on Verdun, thèse de doctorat (histoire), Université McGill, 1997.

30. Mélanie Méthot, L'événement revu : les stratégies de réforme socio-politique proposées par Herbert Brown Ames à Montréal au tournant du XX siècle, 1892-1903, mémoire de maîtrise (histoire), Université de Moncton, 1995.

31. Alain Ruest, Le développement des institutions municipales à Grand-Mère de 1898 à 1925 , mémoire de maîtrise (études québécoises), Université du Québec à Trois-Rivières, 1993.

32. Yannick Gendron, L'émergence de deux municipalités suburbaines de la Mauricie dans l'aprèsguerre: Shawinigan-Sud et Trois-Rivières-Ouest, 1945-1974, mémoire de maîtrise (histoire), Université du Québec à Trois-Rivières, 1998. 
à la bureaucratisation de la municipalité de Montréal de 1900 à 1945. Elle a mis en évidence la dilution progressive de l'emprise des élus sur la gestion, le passage d'une direction paternaliste à une gestion impersonnelle strictement réglementée, et le soutien que chacun des acteurs de l'Hôtel de ville, poursuivant ses propres visées, a apporté au processus de bureaucratisation ${ }^{33}$.

Un deuxième bloc de textes a resserré l'objectif sur l'organisation de services particuliers. Plusieurs ont porté sur les services mis en place par les pouvoirs publics, en général les municipalités : les corps de pompiers montréalais ${ }^{34}$, le traitement de l'eau potable ${ }^{35}$, les loisirs publics au parc de l'île Sainte-Hélène ${ }^{36}$, la justice et les services de police ${ }^{37}$ ont été étudiés. L'auteur de la thèse sur les réformes de la justice pénale à Québec de 1830 à 1860 estime qu'elles s'inscrivaient dans un vaste projet de régulation sociale des masses populaires urbaines, la ville étant considérée comme un lieu de désordre et de dissolution morale et physique. Deux thèses aussi ont examiné la mise en place des services de santé publique. L'une fait ressortir l'autonomie du service de santé de la ville de Montréal vis-à-vis du gouvernement du Québec jusqu’aux années 1970 ${ }^{38}$. L'autre retrace la centralisation et la normalisation graduelles de la gestion sanitaire aux mains d'organismes provinciaux dans les villes de Trois-Rivières et de Shawinigan de 1887 à $1939^{39}$. Son auteur a fait ressortir les résistances des

33. Michèle Dagenais, Dynamiques d'une bureaucratie : l'administration municipale de Montréal et ses fonctionnaires : 1900-1945, thèse de doctorat (histoire), Université du Québec à Montréal, 1992.

34. Bruce D. Redfern, The Montreal Fire Department in the Nineteenth Century : Its Transformation from a Volunteer to a Professional Organization, mémoire de maîtrise (histoire), Université Concordia, 1993.

35. Ginette Gagnon, L'aqueduc de Montréal au tournant du siècle (1890-1914) : l'établissement de la purification de l'eau potable, mémoire de maîtrise (histoire), Université de Montréal, 1999.

36. Josée Desharnais, La gestion des loisirs publics à Montréal : l'exemple du parc de l'île SainteHélène, 1874-1914, mémoire de maîtrise (histoire), Université de Montréal, 1999.

37. Martin Dufresne, La justice pénale et la définition du crime à Québec, 1830-1860, thèse de doctorat (histoire), Université d'Ottawa, 1997; Sylvain Bissonnette, L'intégration des forces policières sur le territoire de la communauté urbaine de Montréal, genèse et évaluation, mémoire de maîtrise (histoire), Université de Montréal, 1993 ; Daniel Dicaire, Police et société à Montréal au milieu du XIX siècle, mémoire de maîtrise (histoire), Université du Québec à Montréal, 1999; Éric Giroux, Les policiers à Montréal : travail et portrait socio-culturel : 1865-1924, mémoire de maitrise (histoire), Université du Québec à Montréal, 1996.

38. Benoît Gaumer, Le service de santé de la ville de Montréal de la mise sur pied au démantèlement, 1865-1975, thèse de doctorat (sciences humaines appliquées), Université de Montréal, 1997.

39. François Guérard, La santé publique dans deux villes du Québec de 1887 à 1939. Trois-Rivières et Shawinigan, thèse de doctorat (histoire), Université du Québec à Montréal, 1993. 
autorités locales à ces processus et a montré comment une large variété d'acteurs sociaux ont interagi dans le domaine de la santé publique.

Du côté des entreprises privées de services, on a examiné l'encadrement juridique du transport en commun ${ }^{40}$, le rôle des Caisses populaires Desjardins dans le développement des quartiers et des banlieues de l'aprèsguerre $^{41}$ ainsi que l'évolution de la téléphonie à Montréal jusqu'en 1930. L'auteure d'une thèse sur ce dernier sujet entend éclaircir les interactions qui s'établissent entre une infrastructure urbaine et son contexte socioculturel. Elle analyse notamment les représentations de la ville et du réseau téléphonique promues par les acteurs privés de la téléphonie et les responsables publics de l'aménagement urbain. Résultat d'une construction sociale, estime-t-elle, l'essor de la téléphonie urbaine a contribué à la reconnaissance d'une nouvelle conception de la ville, celle de la ville réticulée $e^{42}$.

Regroupés, ces travaux sur l'administration et les services urbains forment une mosaïque d'objectifs et d'approches un peu disparate. Plusieurs se rejoignent tout de même en faisant reposer leur analyse sur les rapports de pouvoir entre groupes sociaux ${ }^{43}$. En scrutant les interventions de ceux qui promeuvent la formation d'un milieu urbain conforme à leurs intérêts et perceptions, en éclairant l'émergence et l'action structurante de groupes d'experts de même que la spécialisation accrue des tâches, en dévoilant des jeux complexes d'alliances parfois mouvantes, ils ont fait avancer notre compréhension des mécanismes de la régulation sociale en milieu urbain. Peu d'entre eux, toutefois, ont abordé de front les réactions des autres groupes aux mesures adoptées et l'usage qu'ils font des services urbains, une voie de recherche insuffisamment exploitée.

40. Dany Fougères, L'encadrement juridique des infrastructures et des services publics urbains : le cas du transport en commun à Montréal : 1860-1880, mémoire de maîtrise (histoire), Université du Québec à Montréal, 1991.

41. Clémence Bélanger, Origine et évolution d'une caisse populaire en milieu urbain : le cas de la caisse populaire Sainte-Marguerite de Trois-Rivières (1938-1980), mémoire de maîtrise (études québécoises), Université du Québec à Trois-Rivières, 1997; Jean Poirier, Le crédit dans une caisse populaire en milieu urbain : Saint-Alphonse d'Youville : 1940-1950, mémoire de maîtrise (histoire), Université du Québec à Montréal, 1991.

42. Claire Poitras, La construction des réseaux dans la ville : l'exemple de la téléphonie à Montréal, de 1879 à 1930, thèse de doctorat (aménagement), Université de Montréal, 1997.

43. À noter également, dans cette optique : Élodie Kulesza, Pouvoir et démocratie locale : les élections municipales à Montréal (1960-1974), mémoire de maîtrise (histoire), Université de Rouen, 1996. 


\section{LA VILLE, ENTITÉ PHYSIQUE ET SPATIALE : LA THÉMATIQUE LA MIEUX DÉVELOPPÉE}

Bon nombre des travaux recensés touchent à différents aspects de la ville en tant qu'entité physique et spatiale. Sa morphologie, la différenciation de son espace, la distribution des fonctions urbaines et des citadins sur son territoire, l'aménagement du paysage urbain et l'architecture ont ainsi été abordés. Il s'agit là, en fait, de la thématique la mieux couverte.

Pour le $\mathrm{XvIII}^{\mathrm{e}}$ siècle, quelques textes ont fait sentir à quel point les fortifications de Montréal et de Québec imposent leurs contraintes à l'aménagement ${ }^{44}$ et représentent une césure sociale tout autant que visuelle. Les faubourgs, hors de l'enceinte, sont apparus comme des milieux de vie difficiles, mi-ruraux mi-urbains, à l'architecture modeste et homogène à Québec ${ }^{45}$, et où se concentrent les loyers les plus bas de Montréal. Des thèses de doctorat sur le marché locatif montréalais ${ }^{46}$ et sur les comportements électoraux à Québec ${ }^{47}$ ont souligné l'existence, déjà, d'une différenciation sociospatiale marquée dans la ville préindustrielle. Par contre, à Trois-Rivières, encore étroitement apparentée au milieu rural par ses indicateurs démographiques ${ }^{48}$, l'espace s'est avéré relativement indifférencié, exception faite d'une concentration de commerces sur une rue ${ }^{49}$.

Avec l'industrialisation et l'urbanisation, la différenciation de l'espace urbain s'intensifie et acquiert de nouveaux caractères. Une thèse en géographie montre que l'espace montréalais s'est avéré éminemment malléable dans son ajustement aux besoins de la production manufacturière. Le déploiement des districts industriels, dans certains cas non centraux dès le $\mathrm{xIX}^{\mathrm{e}}$ siècle, apparaît conditionné par les reformulations de l'organisation sociale du travail manufacturier, par les mouvements cycliques de

44. Monique C. Poirier, The Fortifications of Montreal 1717-1744: The Development and Implementation of the Plan, mémoire de maîtrise (art), Université Concordia, 1991.

45. Hélène Bourque, L'architecture domestique des faubourgs Saint-Jean et Saint-Roch avant 1845, mémoire de maîtrise (architecture et urbanisme), Université Laval, 1989.

46. Daniel Massicotte, Montréal et son marché immobilier locatif de 1731 à 1831 : stratification sociale, ségrégation spatiale et transition vers le capitalisme, thèse de doctorat (histoire), Université de Montréal, 1994.

47. David DeBrou, Mass Political Behaviour in Upper-Town Quebec, 1792-1836, thèse de doctorat (histoire), Université d'Ottawa, 1989.

48. Hélène Periers, Une ville à la campagne: Trois-Rivières sous le régime français, profil démographique, mémoire de maîtrise (démographie), Université de Montréal, 1993.

49. Daniel Tremblay, Au cœur du quotidien : la vie matérielle à Trois-Rivières au XVIII siècle, mémoire de maîtrise (sciences humaines/histoire), Université de Sherbrooke, 1993. 
l'investissement industriel et par les interventions de groupes locaux intéressés au développement de tels espaces industriels ${ }^{50}$.

L'espace résidentiel également est remodelé et se différencie selon divers clivages. À Montréal, l'auteur d'un mémoire scrute les origines migratoires des arrivants dans Saint-Louis-du-Mile-End ainsi que leurs caractéristiques de regroupement en cherchant à identifier les forces d'association et d'exclusion qui sculptent le territoire urbain. Il estime que la classe plus que l'ethnie y a orienté les localisations résidentielles ${ }^{51}$. D'autres ont mis l'accent sur les clivages ethniques. Un mémoire sur Westmount, banlieue huppée par excellence de Montréal, montre comment, dans une municipalité jalousement autonome et par une réglementation soignée aussi bien des conduites sociales que de l'usage du sol, une élite anglophone s'est composé un environnement protégé où s'expriment sa différence et ses privi-

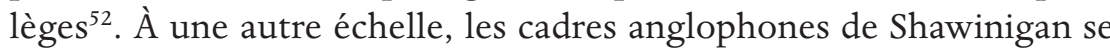
concentrent dans certains secteurs ${ }^{53}$. Il en va de même pour la minorité anglophone de La Tuque qui y aurait imposé un modèle de vie urbaine importé de Nouvelle-Angleterre ${ }^{54}$, et pour les protestants de Québec ${ }^{55}$. Un même phénomène a été observé chez les élus municipaux montréalais dont les investissements fonciers, de surcroît, décalquent la division linguistique de l'espace urbain ${ }^{56}$. Enfin, on a montré que si les ménages montréalais affichent des taux élevés de mobilité résidentielle durant la deuxième moitié $\mathrm{du} \mathrm{xIx}^{\mathrm{e}}$ siècle, les protestants sont moins mobiles que les Irlandais ou les

50. Robert David Lewis, Industry and Space: The Making of Montreal's Industrial Geography, 1850-1918, thèse de doctorat (géographie), Université McGill, 1992.

51. Guy Mongrain, Population et territoire dans un contexte de croissance urbaine : Saint-Louis-duMile-end : 1881-1901, mémoire de maîtrise (histoire), Université du Québec à Montréal, 1998.

52. John Stephen Bryce, The Making of Westmount, Quebec, 1870-1929 : A Study of Landscape and Community Construction, mémoire de maîtrise (géographie), Université McGill, 1990. Voir aussi un mémoire que nous n'avons pu consulter : Roderick MacLeod, Salubrious Settings and Fortunate Families: The Making of Montreal's Golden Square Mile, 1840-1895, thèse de doctorat (histoire), Université McGill, 1997.

53. Marie-Josée Dorion, Cadres et techniciens à Shawinigan, de 1914 à 1945 : évolution sociale d'un groupe professionnel, mémoire de maitrise (études québécoises), Université du Québec à TroisRivières, 1996.

54. Lise Cyr, Un quartier résidentiel de compagnie papetière : la rue Beckler à La Tuque, mémoire de maîtrise (arts et traditions populaires), Université Laval, 1991.

55. Guy Dorval, La géographie de la mortalitéà Québec au XIX siècle : le cas des protestants ensevelis au cimetière Mount Hermon, Sillery, 1843-1883, mémoire de maîtrise (géographie), Université Laval, 1992.

56. Christine Simard, Le personnel politique municipal de Montréal, 1881-1912 : espace urbain, richesse et propriété foncière, mémoire de maitrise (histoire), Université du Québec à Montréal, 1997. 
franco-catholiques ${ }^{57}$. Point à souligner, les déménagements de cette époque s'effectuent généralement sur de courtes distances.

La production de l'environnement construit a aussi retenu l'attention. Un mémoire a dressé un portrait des entrepreneurs en construction au début du siècle à Montréal et montré que, dans un marché dominé par les secteurs industriel et commercial, leurs entreprises font face à une vive concurrence, notamment américaine ${ }^{58}$. Pour expliquer l'évolution de l'habitat, certains auteurs ont mis l'accent sur les caractéristiques des occupants. Ainsi, l'uniformité de l'architecture résidentielle dans deux faubourgs de Québec peuplés majoritairement d'artisans résulterait d'une pratique artisanale tout ancrée dans la tradition jusqu'en 1830 environ ${ }^{59}$. De la même façon, on a attribué à la concentration d'une classe aisée de marchands anglophones et d'hommes de loi francophones les traits architecturaux de la rue d'Auteuil à Québec ainsi que leur stabilité sur une longue période ${ }^{60}$. Une série de facteurs relatifs à la vie familiale, mais aussi à des influences externes, ont été évoqués pour expliquer les réaménagements incessants des maisons de la Cité-Jardin du Tricentenaire à Montréal depuis leur construction vers 1940 par des promoteurs désireux d'édifier une communauté canadienne-française idéale ${ }^{61}$.

D'autres auteurs ont privilégié les grands mouvements de transformation du milieu urbain. Ainsi, le déclin de la maison de fond de cour au $\mathrm{xx}^{\mathrm{e}}$ siècle à Montréal a été attribué notamment aux caractéristiques des nouveaux lotissements, à l'expansion territoriale des activités de centre-ville et à l'exode de la population des quartiers centraux ${ }^{62}$. De même, la détérioration de deux quartiers centraux de Québec sur le plan résidentiel après la Seconde Guerre mondiale a été expliquée soit par l'expansion du centre-ville ${ }^{63}$, soit par la marginalité économique de la région et les maigres

57. Jason Andrew Gilliland, Residential Mobility in Montreal, 1861-1901, mémoire de maitrise (géographie), Université McGill, 1993.

58. Jean-Yves Langlois, Les entrepreneurs en construction à Montréal au début du XXé siècle, mémoire de maîtrise (histoire), Université du Québec à Montréal, 1999.

59. Hélène Bourque, op. cit.

60. Christine Chartré, Évolution historique et architecturale de la rue d'Auteuil, du milieu du XvIII siècle à nos jours, mémoire de maîtrise (histoire de l'art), Université Laval, 1990.

61. Jason Andrew Gilliland, Transforming the Ideal : Visions and Revisions of Housing in a FrenchCanadian Community, 1942-1995, thèse de doctorat (architecture), Université McGill, 1996.

62. Luc Carey, Un type de maison ouvrière : le déclin de la maison de fond de cour à Montréal : 18801920, mémoire de maitrise (histoire), Université du Québec à Montréal, 1996.

63. Pierre Ross, La transformation du quartier Saint-Roch de Québec : 1921-1961, mémoire de maîtrise (histoire), Université Laval, 1989. 
salaires qui en résultent ${ }^{64}$. Divers travaux, en fait, ont tenu compte à la fois de facteurs internes et externes à l'habitat.

Quelques étudiants aussi se sont intéressés à la manipulation symbolique de l'espace urbain. L'organisation des parcs et des terrains de jeux de Montréal, d'après un mémoire, relève pour une bonne part de rapports de pouvoir entre différents groupes de la société montréalaise, et, sur un plan idéologique, est imprégnée de considérations relatives au genre, à la classe sociale, à l'ethnie et à la sexualité ${ }^{65}$. L'évolution récente des formes architecturales à Québec a été reliée à celle des idéologies ${ }^{66}$. Un archéologue, aussi, a recherché, pour chaque phase de l'évolution d'un site à Québec pendant trois siècles, le paradigme qui fonde l'organisation physique et conceptuelle des aménagements ${ }^{67}$.

Ils sont donc nombreux les étudiants qui, prolongeant une tradition déjà bien ancrée, ont conféré à la dimension spatiale et physique de la ville un rôle significatif dans leur démarche. Il s'agit là en fait d'une ligne de force de l'histoire urbaine au Québec. Elle y témoigne d'une certaine vigueur de la géographie historique, en même temps que du maintien de son intérêt pour les questions urbaines. Après l'histoire, la géographie est d'ailleurs la discipline la mieux représentée dans le corpus : $63 \%$ des travaux retenus proviennent de programmes d'histoire, $10 \%$ de géographie, $10 \%$ des études québécoises (un programme interdisciplinaire où la géographie historique occupe précisément une place importante) et le reste est dispersé parmi plusieurs programmes (arts et traditions populaires, histoire de l'art, architecture, urbanisme...).

Ce mariage de l'histoire et de la géographie est également perceptible dans les codirections de mémoires et de thèses. En effet, la majorité des rencontres interdisciplinaires en codirection, soit neuf sur douze ${ }^{68}$, ont été celles d'historiens et de géographes. On le voit, n'était de ces codirections, trois étudiants seulement auraient été supervisés par des chercheurs issus

64. Anne-Marie Séguin, Un quartier à l'ombre de l'État : transformations sociales et spatiales du quartier Saint-Jean-Baptiste de Québec, de 1950 à nos jours, thèse de doctorat (géographie), Université Laval, 1990.

65. Sarah Schmidt, Domesticating Parks and Mastering Playgrounds: Sexuality, Power and Place in Montreal, 1870-1930, mémoire de maîtrise (histoire), Université McGill, 1996.

66. Nathalie Thibault, Formes architecturales et idéologies à Québec, 1960-1990, mémoire de maîtrise (géographie), Université Laval, 1990.

67. William Moss, Une archéologie du paysage urbain : la Terrasse Dufferin à Québec, du XVII ${ }^{e}$ au XX siècles, mémoire de maîtrise (arts et traditions populaires), Université Laval, 1990.

68. Onze thèses sur vingt et treize mémoires sur cinquante ont été supervisés en codirection, soit environ le tiers des travaux. 
de plus d'une discipline, ce qui étonne : de nombreux spécialistes de l'histoire urbaine ne se sont-ils pas faits les défenseurs d'une plus large ouverture aux pratiques interdisciplinaires ${ }^{69}$ ? Souhaitons que cet heureux concours de l'histoire et de la géographie inspire un plus vaste déploiement de telles pratiques dans le champ de l'histoire urbaine. Au vu des résultats de recherche des étudiants qui se sont souciés de la ville comme entité physique, cela ne pourrait que mener à un enrichissement : des travaux féconds ont été livrés par ceux qui se sont interrogés sur l'intime imbrication du social et du spatial, qui ont fait porter leur analyse sur différentes échelles et qui ont situé leur sujet dans des dynamiques larges.

\section{SOCIÉTÉ ET CULTURE URBAINES :} LA QUESTION DE LA MODERNITÉ

La dernière classe thématique retenue, la moins clairement délimitée aussi, regroupe des travaux qui apportent un éclairage sur divers aspects de la société et de la culture urbaines.

Certains textes ont alimenté les débats sur la nature de la société urbaine au Québec et sur sa propension à la tradition ou à la modernité. Un mémoire argumente qu'à Shawinigan jusqu'en 1930, les curés et leurs paroissiens issus des campagnes proches transposent en milieu urbain une culture traditionnelle empreinte d'ultramontanisme ${ }^{70}$. À Montréal, une thèse a donné une tout autre image de la paroisse Saint-Pierre-Apôtre en la présentant comme un outil d'intégration à une vie sociale et urbaine résolument moderne, à travers ses œuvres et organismes. Ce rôle s'érode après la Première Guerre mondiale, alors que les institutions purement locales sont en partie supplantées par des organismes supraparoissiaux ${ }^{71}$. Une autre thèse montre que l'un de ces organismes a suivi durant les an-

69. Voir, par exemple, Theodore Hershberg, "The New Urban History : Toward an Interdisciplinary History of the City ", Journal of Urban History, 5,1 (1978) : 3-40. Pour la France, Bernard Lepetit et Jean-Luc Pinol soulignent que l'histoire urbaine est apparue à plusieurs comme le lieu où pourrait, du moins en partie, être pratiquée une histoire qui intégrerait diverses disciplines en vue d'en arriver à une vision globalisante, celle de l'histoire «totale». Voir B. Lepetit et J.-L. Pinol, "France», dans Richard Rodger, dir., European Urban History. Prospect and Retrospect (Leicester, Leicester University Press, 1993), 83.

70. René Bergeron, Encadrement clérical en contexte d'urbanisation à Shawinigan, 1908-1930, mémoire de maîtrise (études québécoises), Université du Québec à Trois-Rivières, 1997.

71. Lucia Ferretti, La société paroissiale en milieu urbain : Saint-Pierre-Apôtre de Montréal : 18481930, thèse de doctorat (histoire), Université du Québec à Montréal, 1990. Voir aussi deux mémoires que nous n'avons pas eus en main : Jean-Christian Aubry, Pratiques religieuses et vie paroissiale en milieu urbain au Xx ${ }^{e}$ siècle. La paroisse Immaculée-Conception de Montréal, 1910-1940, mémoire 
nées 1940 et 1950 un parcours qui va aussi de l'innovation au repli : la Ligue ouvrière catholique canadienne, après avoir inscrit ses interventions en matière d'habitation et d'urbanisme dans le cadre d'une action sociale catholique axée sur la ville et les ouvriers, se retranche en effet dans des positions plus traditionnelles ${ }^{72}$.

Nos connaissances sur la société et la culture urbaines s'enrichissent aussi de textes sur le monde des arts dont on a longtemps dit qu'ils avaient tardé à représenter la ville avant 1940 . Or on apprend que le roman urbain apparaît bien plus tôt, sans toutefois dépeindre la vie des petites gens comme le roman réaliste qui va suivre ${ }^{73}$. Mais on apprend aussi que pareil réalisme émerge tardivement dans la peinture de paysages urbains durant les années 1930, comme l'illustre l'œuvre charnière d'un artiste montréalais habité par des préoccupations profondes pour les problèmes sociaux ${ }^{74}$.

Quelques textes sur les arts de la scène $\mathrm{e}^{75}$ les montrent beaucoup moins repliés sur la tradition qu'on l'avait affirmé précédemment. Le théâtre lyrique à Montréal de 1840 à la Première Guerre mondiale comme le théâtre à Québec au début du siècle sont marqués par leur intégration à l'industrie nord-américaine du spectacle, et ce particulièrement chez les anglophones, mieux organisés et desservis. À partir de la fin du XIX ${ }^{\mathrm{e}}$ siècle, un théâtre francophone s'organise cependant qui jouit d'un certain succès auprès de la population ouvrière. On a argumenté que durant l'entredeux-guerres, le théâtre burlesque populaire de langue française véhiculait la voix des masses en exprimant l'américanité de la culture urbaine québécoise, au grand dam des élites francophones ${ }^{76}$.

de maîtrise (histoire), Université de Montréal, 1998; Steve Roussel, Encadrement religieux et vie associative dans une paroisse ouvrière : Sainte-Jeanne-d'Arc de Sherbrooke de 1921 à 1952, mémoire de maîtrise (sciences humaines/histoire), Université de Sherbrooke, 1998.

72. Jean-Pierre Collin, La ligue ouvrière catholique canadienne, 1938-1965 : un mouvement social urbain, thèse de doctorat (histoire), Université du Québec à Montréal, 1990.

73. Isabelle Gélinas, L'image de la ville dans la prose narrative québécoise, 1914-1936, mémoire de maitrise (histoire), Université de Montréal, 1994.

74. Monique Nadeau-Saumier, Louis Muhlstock: The Urban Landscape, 1930-1950, mémoire de maîtrise (histoire de l'art), Université Concordia, 1989.

75. Mireille Barrière, La société canadienne-française et le théâtre lyrique à Montréal entre 1840 et 1913, thèse de doctorat (histoire), Université Laval, 1990; Christian Beaucage, Pour une histoire sociologique du théatre : l'activité théâtrale francophone à Québec de 1900 à 1911, thèse de doctorat (littérature canadienne-française), Université Laval, 1993.

76. Donald Cuccioletta, The Américanité of Quebec Urban Popular Culture as Seen Through Burlesque Theater in Montreal (1919-1939), thèse de doctorat (histoire), Université du Québec à Montréal, 1997. 
Enfin, peu de textes s'attaquent spécifiquement aux modes de vie urbains. Deux mémoires tout de même portent sur la vie matérielle à TroisRivières au $\mathrm{xVIII}^{\mathrm{e}}$ siècle ${ }^{77}$ et sur la composition des régimes alimentaires à Montréal au début du $\mathrm{XIX}^{\mathrm{e}}$ siècle, laquelle paraît déterminée plus par la classe sociale que par l'ethnie ou les préférences individuelles ${ }^{78}$. Deux thèses, aussi, ouvrent des perspectives intéressantes notamment sur la sociabilité urbaine ${ }^{79}$. L'une porte sur les petits commerçants de l'alimentation à Montréa $1^{80}$ et, par le biais du crédit principalement, sur les rapports qu'ils entretiennent avec les milieux populaires montréalais de 1920 à 1940. L'autre explore les effets de l'industrialisation sur la reproduction humaine et dévoile différentes stratégies des ménages de Saint-Hyacinthe tels les voisinages entre parents et les cohabitations ${ }^{81}$. Un mémoire, enfin, aborde des questions démographiques dans cette ville ${ }^{82}$.

\section{L'ÉLARGISSEMENT DES PERSPECTIVES}

Il y a quinze ans, Paul-André Linteau et Alan Artibise émettaient toute une série de suggestions pour les recherches à venir en histoire urbaine au Canada $^{83}$. Plusieurs ont été suivies, ce dont témoigne par exemple l'étendue du spectre thématique couvert par les étudiants. D’autres recommandations, parmi les plus fondamentales, ont eu peu d'échos. Il en va ainsi de l'adoption de territoires d'enquête élargis et de l'intensification souhaitée des pratiques interdisciplinaires, lesquelles devaient contribuer à consolider le substrat théorique des études historiques urbaines.

En fait, l'amplitude non seulement des territoires, mais aussi, faut-il ajouter, des périodes d'enquête s'avère assez restreinte. On s'est appliqué

77. Daniel Tremblay, op. cit.

78. Donald William Fyson, Eating in the City : Diet and Provisioning in Early Nineteenth-Century Montreal, mémoire de maîtrise (histoire), Université McGill, 1989.

79. Un mémoire de maîtrise aborde aussi la sociabilité urbaine : Juliette LePlomb, Les sociabilités urbaines entre tradition et innovations à Montréal au début du Xxe siècle, mémoire de maîtrise (histoire), Université Lumière (Lyon), 1997.

80. Sylvie Taschereau, Les petits commerçants de l'alimentation et les milieux populaires montréalais, 1920-1940, thèse de doctorat (histoire), Université du Québec à Montréal, 1992.

81. Peter Gossage, Famille et population dans une ville manufacturière : Saint-Hyacinthe : 18541914/Family and Population in a Manufacturing Town : Saint-Hyacinthe : 1854-1914, thèse de doctorat (histoire), Université du Québec à Montréal, 1991.

82. Liette Côté, La vieillesse au Québec au XIX siècle : le cas de Saint-Hyacinthe, 1861-1891, mémoire de maîtrise (histoire et sciences politiques), Université de Sherbrooke, 1998. Nous n'avons pas eu ce texte en main.

83. P.-A. Linteau et A. F. J. Artibise, op. cit. 
à démonter pièce par pièce la mécanique des transformations urbaines à l'aide d'études de cas. Ce travail campe des assises solides aux réflexions des chercheurs. Mais il serait souhaitable que l'ambition intellectuelle des étudiants de doctorat porte certains d'entre eux vers des questions plus englobantes dans le temps ou l'espace ${ }^{84}$. Voici trois exemples d'insuffisances à cet égard : le réseau urbain québécois est ignoré; il est rare qu’un sujet soit abordé dans plus d'une ville à la fois en vue de dépasser les spécificités locales; les deux univers urbains sis de part et d'autre d'une lignefrontière tirée en 1800 ne sont affrontés qu'isolément par les historiens.

Le grand projet de l'histoire urbaine, perçue comme un lieu idéal de rencontre et d'harmonisation des perspectives de recherche issues de diverses disciplines, n'a pas laissé de marque profonde dans la production des étudiants de maîtrise et de doctorat. D’une part, des disciplines comme la démographie et la sociologie sont fort peu représentées, nous privant d'éclairages essentiels. D'autre part, exception faite de l'histoire et de la géographie, les fossés interdisciplinaires n’ont été que peu enjambés. Certes, un début d'ouverture aux acquis historiens sur la société québécoise se manifeste en histoire de l'art et de l'architecture, de même qu'en archéologie; plusieurs étudiants historiens ont aussi puisé en partie leur inspiration dans des disciplines autres que la leur, avec des résultats intéressants. Malgré ces signes prometteurs, le dialogue interdisciplinaire demeure, nous semble-t-il, assez ténu, sans quoi il se serait notamment concrétisé dans des codirections plus nombreuses.

Le tableau brossé ici ne tient compte que des travaux étudiants. Y ajouter les travaux publiés en aurait sans doute modifié quelque peu la configuration, peut-être en augmentant la part des travaux portant sur des périodes plus longues ou des territoires plus vastes. Il traduit tout de même assez bien les préoccupations, les pratiques et les approches qui ont dominé le champ de l'histoire urbaine depuis une dizaine d'années.

84. Le Québec n'est pas le seul à laisser de côté les études globalisantes, comme le fait remarquer Jean-Luc Pinol pour l'Angleterre : «Telle est d'ailleurs la tendance actuelle des historiens britanniques qui n'envisagent plus d'études urbaines à ambition globalisante mais qui concentrent leurs efforts sur des aspects spécifiques de l'histoire urbaine. " Jean-Luc Pinol, Le monde des villes au XIX ${ }^{e}$ siècle (Paris, Hachette, 1991, 5). Charles Tilly abonde en ce sens, pour l'histoire urbaine en général : «Urban historians, for the most part, love particulars and fear grand schemes.» Charles Tilly, «What Good is Urban History?», Journal of Urban History, 22,6 (septembre 1996) : 715. 